\title{
Targeting XPO1 enhances innate immune response and inhibits KSHV lytic replication during primary infection by nuclear stabilization of the p62 autophagy adaptor protein
}

\author{
Wen Meng ${ }^{1}$ and Shou-Jiang Gao
}

\begin{abstract}
Nucleocytoplasmic transport of signaling modulators is essential for regulating cellular responses to extracellular stimulation and stress, as well as pathogen infection. Exportin 1 (XPO1), also known as chromosomal maintenance 1 (CRM1), mediates nuclear export of proteins, rRNAs, snRNAs, and some mRNAs. In this study, we have identified an essential role of XPO1 in regulating Kaposi's sarcoma-associated herpesvirus (KSHV) lytic replication during primary infection of primary human umbilical vein endothelial cells. Treatment with an XPO1 inhibitor KPT-8602 and short hairpin RNA (shRNA)-mediated knockdown of XPO1 reduced KSHV lytic replication but had no effect on KSHV entry and trafficking. XPO1 inhibition induced retention of autophagy adaptor protein p62 (SQSTM1) in the nucleus, which enhanced activation of TBK1 and IRF3. As a result, nuclear accumulation of p62 increased expression of innate immune-related genes including IRF7, ISG15, IFIT1, IFIT2, and IFIT3, leading to a reduction of KSHV Iytic replication. These results illustrate a novel mechanism by which XPO1 mediates innate immune response and KSHV replication, and identify XPO1 as a potential therapeutic target and KPT-8602 as a promising therapeutic agent for KSHV infection.
\end{abstract}

\section{Introduction}

Kaposi's sarcoma-associated herpesvirus (KSHV) is a gammaherpesvirus etiologically associated with Kaposi's sarcoma and several other lymphoproliferative diseases ${ }^{1-3}$. KSHV has both latent and lytic replication cycles regulated by intracellular and extracellular signals ${ }^{4}$. In lytic replication-permissive cells such as primary human umbilical vein endothelial (HUVEC), activation of ERK, p38, and JNK pathways promotes expression of viral lytic genes, and KSHV undergoes robust lytic replication before entering into latency ${ }^{5-8}$. However, activation of $\mathrm{NF}-\mathrm{kB}$ pathway promotes the expression of latent genes and KSHV enters into latency in nonpermissive cells such

Correspondence: Shou-Jiang Gao (gaos8@upmc.edu)

'UPMC Hillman Cancer Center, Department of Microbiology and Molecular Genetics, University of Pittsburgh, Pittsburgh, PA, USA

Edited by H.-U. Simon as primary human dermal microvascular endothelial cells and human foreskin fibroblasts ${ }^{9-12}$.

The exchange of signaling molecules between nuclear and cytoplasmic compartments is essential for cellular functions. This process is mediated by a nuclear pore complex (NPC) inserted across the nuclear envelope (NE). Unlike smaller molecules, which diffuse through NPCs, the shuttling of proteins larger than $40 \mathrm{kDa}$ is regulated by karyopherins (Kaps), which interact with NPC components nucleoporins (Nups) to cross $\mathrm{NE}^{13}$.

A Kap consists of a cargo-binding domain, an NPCbinding domain, and an N-terminal Ras-related nuclear protein (Ran) binding domain ${ }^{14}$. Ran is a small GTPase, switching between a cytoplasmic RanGDP form and a nuclear RanGTP form by nucleotide exchange and GTP hydrolysis. Kaps include both importins and exportins. For import, after importins transport cargo molecules through NPCs into the nucleus, RanGTP stimulates

\section{(c) The Author(s) 2021}

(c) Open Access This article is licensed under a Creative Commons Attribution 4.0 International License, which permits use, sharing, adaptation, distribution and reproduction cc) in any medium or format, as long as you give appropriate credit to the original author(s) and the source, provide a link to the Creative Commons license, and indicate if changes were made. The images or other third party material in this article are included in the article's Creative Commons license, unless indicated otherwise in a credit line to the material. If material is not included in the article's Creative Commons license and your intended use is not permitted by statutory regulation or exceeds the permitted use, you will need to obtain permission directly from the copyright holder. To view a copy of this license, visit http://creativecommons.org/licenses/by/4.0/. 
dissociation of the importin-cargo complex to release cargos $^{15}$. For export, exportin-cargo complex formation is induced by RanGTP. After transportation into the cytoplasm, hydrolysis of GTP to GDP results in a switch from RanGTP to RanGDP to release cargos ${ }^{16}$.

There are seven members of exportin, including XPO1 (CRM1), XPO2 (CSE1L), XPO3 (XPOt), XPO4, XPO5, $\mathrm{XPO} 6$, and $\mathrm{XPO}^{13}{ }^{13} \mathrm{XPO} 1, \mathrm{XPO} 2, \mathrm{XPO} 4, \mathrm{XPO}$, and $\mathrm{XPO} 7$ primarily mediate the export of proteins, whereas $\mathrm{XPO} 3$ and $\mathrm{XPO} 5$ are involved in the export of tRNAs and precursor microRNAs, respectively. XPO1 is the major exportin that mediates protein nuclear export, which is important for the replication of several viruses including retroviruses, orthomyxoviruses, paramyxoviruses, flaviviruses, coronaviruses, rhabdoviruses, and herpesviruses ${ }^{17,18}$. Hence, XPO1 is a potential target for antiviral therapy.

XPO1 transports proteins containing leucine-rich nuclear export signals (NESs) ${ }^{19,20}$. A novel class of selective inhibitor of nuclear export (SINE) compounds that bind to XPO1 NES groove was identified ${ }^{21-23}$. Unlike leptomycin B (LMB), SINE compounds form a reversible covalent bond with XPO1 cysteine 528, making it less cytotoxic than LMB to cells. KPT-8602 (Eltanexor) is a second-generation SINE with reduced penetration across blood-brain barrier and an enhanced tolerability profile ${ }^{24}$.

Here, we have identified a critical role of XPO1 in KSHV lytic replication during primary infection. We show that XPO1 inhibition with KPT-8602 and by shRNA knockdown significantly reduces infectious virions by inhibiting viral gene expression. XPO1 inhibition induces p62 nuclear retention, which blocks KSHV lytic replication by activating TBK1 and IRF3 and enhancing the expression of innate immune-related genes. These results identify XPO1 as an essential protein for KSHV lytic replication by regulating p62 nuclear shuttling and controlling antiviral immune responses.

\section{Materials and methods \\ Cells and drug}

HUVEC (ATCC) was cultured in VascuLife VEGF complete medium (Lifeline). KSHV-infected iSLK (iSLKBAC16) cells were from Dr. Jae Jung and cultured as previously described ${ }^{25}$. KPT-8602 (Selleck) was dissolved in dimethyl sulfoxide (DMSO).

\section{Virus preparation and titration}

KSHV was prepared from iSLK-BAC16 cells as previously described ${ }^{25}$. KSHV in VascuLife VEGF complete medium without Heparin was titrated by infecting HUVEC as previously described ${ }^{5,26}$.

\section{Virus infection}

HUVEC were infected with KSHV at a multiplicity of infection of two IUs per cell ${ }^{5,26}$. To determine KPT-8602's effect, HUVEC were pretreated with KPT-8602 or vehicle control DMSO for $1 \mathrm{~h}$. Cells were then infected with KSHV with KPT-8602 or vehicle. At $4 \mathrm{~h}$ post infection (hpi), the medium was replaced with fresh medium. Cells were fixed at 6 hpi for examining virus entry and trafficking, at $24 \mathrm{hpi}$ for examining viral infectivity, and at 48 hpi for examining viral transcripts and proteins. To examine infectious virions, supernatants were collected at 96 hpi and titrated ${ }^{5,26}$.

\section{Immunofluorescence assay}

Immunofluorescence assay was performed as previously described $^{5}$ using antibodies to ORF65 ${ }^{5}$, LANA (Abcam), p62 (Cell Signaling), phosphorylated IRF3 (pIRF3, Novus Biologicals), and pTBK1 (Cell Signaling). Signal was detected with an Alexa488-, Alexa555-, or Alexa647conjugated secondary antibody (Life Technologies). Images were acquired using an Olympus FV2000 Confocal Microscope with CellSens Software. Magenta of Alexa647 was shown as green pseudocolor for better presentation.

\section{Western blotting}

Western blotting was performed as previously described $^{27}$ using primary antibodies against LANA (Abcam), K-bZIP (Santa Cruz), $\beta$-actin (Santa Cruz), XPO1 (Sigma), p62 (Cell Signaling), pTBK1 (Cell Signaling), TBK1 (Novus Biologicals), pIRF3 (Novus), IRF3 (Novus), STING (Novus), and LC3B (Sigma). ORF65 and RTA antibodies were previously described ${ }^{5,28}$.

\section{RNA extraction and RT-qPCR}

Expression of viral genes was analyzed by reverse transcription-quantitative polymerase chain reaction (RTqPCR) as previously described ${ }^{8}$. qPCR primers were listed in Supplementary Table 1. Three biological repeats were performed for each treatment.

\section{DNA extraction}

DNA extraction was performed by using QIAamp DNA Mini Kit (Qiagen).

\section{shRNA knockdown}

Lentiviral production and knockdown with shRNAs were performed as previously described ${ }^{29}$. Supernatants were collected $72 \mathrm{~h}$ after transfection. HUVEC were infected with lentiviruses by spinning infection at $500 \times g$ in $10 \mu \mathrm{g} / \mathrm{ml}$ Polybrene (Sigma). The effect of shRNA knockdown was examined at $48 \mathrm{hpi}$.

\section{Statistical analysis}

Results are expressed as means and standard deviations. Statistical analyses were performed using Prism 5.01 (GraphPad Software). Significance was determined by twoway ANOVA followed by Tukey's multiple comparisons test. 


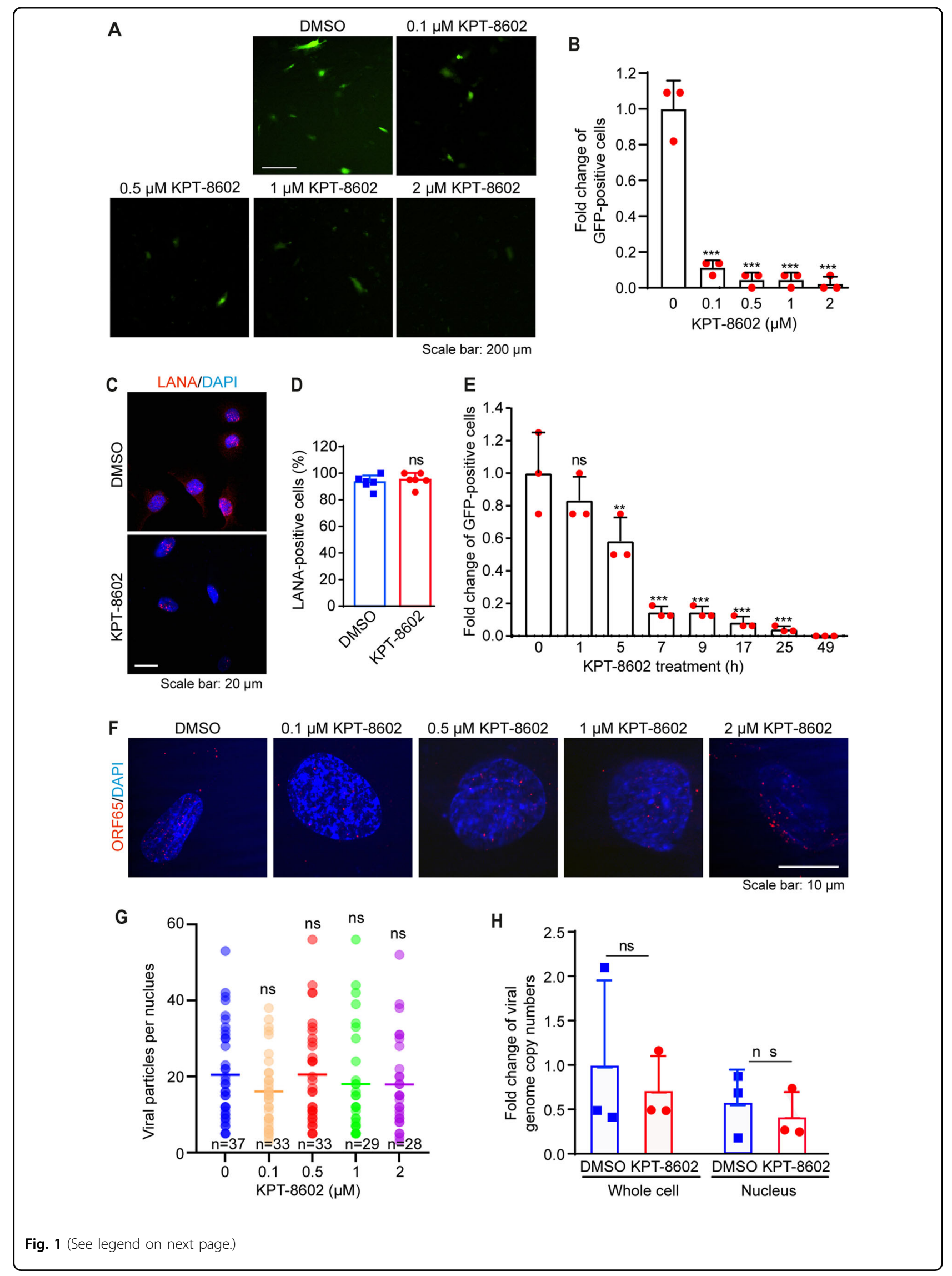


(see figure on previous page)

Fig. 1 XPO1 inhibitor KPT-8602 decreases the production of KSHV infectious virions but has no effect on KSHV infectivity, and virus entry and trafficking during primary infection. A, B HUVEC were pretreated with the indicated doses of KPT-8602 for $1 \mathrm{~h}$ and then infected with KSHV in the presence of KPT-8602. At 4 hpi, cells were extensively washed and replaced with a new medium containing the indicated concentrations of KPT8602. At $48 \mathrm{hpi}$, cells were extensively washed and replaced with a new medium without KPT-8602. Supernatants were collected at $96 \mathrm{hpi}$ and used to titrate infectious virions by infecting fresh HUVEC. At 48 hpi, representative images were taken (A) to quantify the number of GFP-positive cells (B). C HUVEC pretreated with $0.5 \mu \mathrm{M}$ KPT-8602 for $1 \mathrm{~h}$ were infected with KSHV in the presence of KPT-8602. At 48 hpi, cells were fixed and stained for LANA (red). The expression level and staining pattern of LANA are shown. Nuclei were stained with DAPI. Images were taken with confocal microscopy (magnification $\times 600$ ). D Quantification of LANA-positive cells based on five images taken as described in (C). E HUVEC were pretreated with $0.5 \mu \mathrm{M}$ KPT-8602 for $1 \mathrm{~h}$ and then infected with KSHV in the presence of KPT-8602 for the indicated times. Cells were extensively washed and replaced with a new medium without KPT-8602. At 50 hpi, cells were replaced with the new medium. Supernatants were collected at 96 hpi and used to titrate infectious virions by infecting fresh HUVEC. At 48 hpi, GFP-positive cells were quantified. F HUVEC pretreated with $0.5 \mu M$ KPT-8602 for $1 \mathrm{~h}$ were infected with KSHV in the presence of KPT-8602 for $6 \mathrm{~h}$, fixed, and stained for nuclei with DAPI (blue) and KSHV particles with an antibody against ORF65 (red). Images were taken with a confocal microscopy (magnification $\times 1000$ ). G The total number of KSHV particles successfully docked at the perinuclear region was determined and analyzed based on images taken as described in (F). H HUVEC pretreated with $0.5 \mu \mathrm{M} \mathrm{KPT-8602} \mathrm{for} 1 \mathrm{~h}$ were infected with KSHV in the presence of KPT-8602. Cells were harvested at 6 hpi. Cellular DNA and viral DNA were extracted from whole cells or the nuclear fraction for the detection of the viral genome by qPCR using LANA-specific primers. ${ }^{*}$, **, and *** indicate $P$ values of $<0.05,<0.01$, and $<0.001$, respectively; NS not significant.

\section{Ethics statement}

All authors had access to all data and have reviewed and approved the final paper.

\section{Results}

\section{XPO1 inhibitor KPT-8602 decreases KSHV titer}

We used KPT-8602 to investigate XPO1's role in KSHV primary infection in HUVEC as these cells support productive infection ${ }^{5}$. HUVEC treated with KPT-8602 for $1 \mathrm{~h}$ were infected with KSHV. At $48 \mathrm{hpi}$, cells were replaced with fresh medium without KPT-8602. At 96 hpi, supernatants were collected and titrated. KPT-8602 reduced KSHV titer at a dose-dependent fashion (Fig. 1A, B). KPT8602 reduced titers by $84 \%$ at $0.1 \mu \mathrm{M}$ and by $98 \%$ at $2 \mu \mathrm{M}$. At $0.1 \mu \mathrm{M}, \mathrm{KPT}-8602 \mathrm{had}<30 \%$ cytotoxic effect on cells before $48 \mathrm{~h}$, and $>40 \%$ of the cells remained alive at 72 and $96 \mathrm{~h}$ (Supplementary Fig. 1). At $2 \mu \mathrm{M},>45 \%$ of the cells remained viable at any points examined. Thus, KPT8602's inhibitory effect on the production of KSHV virions was unlikely due to its cell cytotoxicity.

\section{KPT-8602 inhibits KSHV lytic replication at post-entry stage(s)}

To identify step(s) of KSHV infection regulated by XPO1, we examined whether XPO1 inhibition might affect infectivity. We used $0.5 \mu \mathrm{M}$ KPT-8602 based on the cytotoxicity results (Supplementary Fig. 1). Over $70 \%$ of cells survived at 24 and $48 \mathrm{~h}$, and $>40 \%$ of cells survived at 72 and $96 \mathrm{~h}$. HUVEC pretreated with DMSO or $0.5 \mu \mathrm{M}$ KPT-8602 for $1 \mathrm{~h}$ were infected with KSHV. At $4 \mathrm{hpi}$, cells were replaced with fresh medium without the inhibitor. At $48 \mathrm{hpi}$, cells were stained for LANA protein. Detection of LANA protein indicated successful viral infection. KPT-8602 treatment did not affect the percentage of LANA-positive cells (Fig. 1C, D), LANA staining pattern and intensity, and the number of LANA punctate dots per cell (Fig. 1C). Hence, KPT-8602 neither affected KSHV infectivity nor LANA protein expression. To confirm that KPT-8602 was unlikely to affect KSHV entry and trafficking, we treated HUVEC with $0.5 \mu \mathrm{M} \mathrm{KPT-8602} \mathrm{for} 1 \mathrm{~h}$ and then infected them with KSHV. Cells were replaced with fresh medium without the inhibitor at the indicated time points. Supernatants were titrated for infectious virions at $96 \mathrm{hpi}$. KPT-8602 treatment for $1 \mathrm{~h}$ had no significant effect on KSHV titer (Fig. 1E). However, KPT8602 treatment for $>5$ h significantly reduced KSHV titer in a time-dependent fashion (Fig. 1E). Particularly, KPT8602 treatment for $>7 \mathrm{~h}$ reduced KSHV titer by $84 \%$. These results confirmed that KPT-8602 was unlikely to affect the early steps of KSHV infection.

We then investigated whether KPT-8602 affected the trafficking of KSHV particles to the nucleus. HUVEC pretreated with DMSO or KPT-8602 for $1 \mathrm{~h}$ were infected with KSHV for $6 \mathrm{~h}$ and stained for ORF65 protein to directly visualize viral capsids (Fig. 1F) ${ }^{30}$. KPT-8602 treatment did not affect the numbers of viral particles reaching the nucleus (Fig. 1F, G). We further examined KSHV genomes injected into the nucleus by qPCR. HUVEC pretreated with DMSO or $0.5 \mu \mathrm{M}$ KPT8602 for $1 \mathrm{~h}$ were infected with KSHV with KPT-8602. At $6 \mathrm{hpi}$, the nuclear and cytoplasmic separation was performed, and both cellular and viral DNA were extracted and quantified by qPCR. XPO1 inhibition did not affect the numbers of viral genomes in cells and in the nucleus (Fig. 1H). These results confirmed that KPT8602 had no effect on virus entry and trafficking. Thus, KPT-8602 likely reduced KSHV virion production at a post entry stage.

\section{KPT-8602 inhibits expression of KSHV lytic genes}

KSHV lytic genes include immediate-early (IE), early $(\mathrm{E})$, and late (L) genes, whereas latent genes mainly consist of LANA, vFLIP, vCyclin, ORF-K12, and a cluster of 
microRNAs ${ }^{4}$. During KSHV primary infection in HUVEC, latent transcripts expressed first, followed by lytic transcripts in the order of IE, E, and L transcripts, which peaked at around $54 \mathrm{hpi}^{8}$. We monitored the kinetics of KSHV lytic genes by RT-qPCR. KPT-8602 significantly reduced mRNA levels of lytic genes including RTA (ORF50), ORF45, vIL-6 (ORF-K2), MTA (ORF57), ORF59, ORF65, and ORF-K8.1 (Fig. 2A-H). For KSHV latent genes, KPT-8602 treatment also inhibited the expression of vIRF3 and ORF-K12 (Fig. 2I, J) but had no significant effect on vCyclin and LANA (Fig. 2K, L). The Western-blotting analysis confirmed that KPT8602 treatment reduced the expression of lytic proteins RTA, K-bZIP (ORF-K8), and ORF65 but only had a marginal effect on latent protein LANA (Fig. 2M), which was consistent with LANA's immunostaining results (Fig. 1C, D). These results demonstrated that XPO1 had an essential role in the expression of KSHV lytic genes.

\section{XPO1 knockdown inhibits KSHV lytic replication}

To confirm KPT-8602's inhibitory effect on KSHV replication, we performed shRNA knockdown of XPO1. $\mathrm{XPO} 1$ protein was reduced by $>80 \%$ in HUVEC transduced with lentiviruses expressing XPO1 shRNAs (Fig. 3A). XPO1 knockdown significantly reduced infectious virions by $80 \%$ compared to the scrambled control (Fig. 3B, C), confirming KPT-8602's effect and XPO1's critical role in KSHV replication.

To confirm the step of KSHV replication regulated by XPO1, we determined the effect of XPO1 knockdown on KSHV infectivity. HUVEC with XPO1 knockdown were infected with KSHV and stained for LANA protein at 48 hpi. XPO1 knockdown did not change the percentage of LANA-positive cells (Fig. 3D, E), and LANA staining pattern and intensity (Fig. 3D), indicating that XPO1 neither regulated KSHV infectivity nor LANA protein expression.

We further examined the effect of XPO1 knockdown on KSHV entry and trafficking. HUVEC with XPO1 knockdown were infected with KSHV for $6 \mathrm{~h}$ and stained for ORF65 protein (Fig. 3F). There was no difference in virus particles docked at the perinuclear region between cells transduced with XPO1 shRNAs or scrambled control (Fig. 3G). XPO1 knockdown also did not affect the internalization of the viral genome into cells and the nucleus (Fig. 3H). These results confirmed that XPO1 did not regulate virus entry and trafficking.

Finally, we investigated the effect of XPO1 knockdown on the expression of KSHV genes. HUVEC with XPO1 knockdown were infected with KSHV for $48 \mathrm{~h}$. XPO1 knockdown significantly reduced mRNA levels of lytic genes RTA, ORF57, and ORF65 (Fig. 3I-K) but only had a minor effect on latent gene LANA (Fig. 3L). XPO1 knockdown also significantly decreased lytic proteins
K-bZIP and ORF65 but not latent protein LANA (Fig. 3M). These results confirmed XPO1's essential role in KSHV lytic replication during primary infection.

\section{KPT- 8602 retains $p 62$ in the nucleus and enhances IRF3 and TBK1 activation}

We have previously shown that p62 protein is retained in the nucleus in AGS and HUH7 cells following KPT8602 treatment $^{31}$. KPT-8602 treatment upregulated p62 protein level and retained p62 in nucleus of uninfected HUVEC (Supplementary Fig. 2). KSHV infection only had a marginal effect on the p62 level.

It was reported that cytosolic p62 could attenuate the cGAS-STING pathway and RIG-mediated type I interferon (IFN) signaling ${ }^{32,33}$. We investigated the activation of TBK-1 and IRF3 upon KPT-8602 treatment. HUVEC were pretreated with $0.5 \mu \mathrm{M}$ of KPT-8602 or DMSO for $1 \mathrm{~h}$, and then mock-infected or infected with KSHV. At 12 hpi, KSHV infection slightly activated both TBK-1 and IRF3 (Fig. 4A, B) as previously reported ${ }^{34,35}$. While infection by a DNA virus can activate an innate immune response, KSHV encodes numerous genes that antagonize this response ${ }^{34,36-40}$. KPT-8602 treatment alone was sufficient to increase levels of both pIRF3 and pTBK1 (Fig. 4A, B). Hence, KPT-8602-induced p62 nuclear accumulation and stabilization might lead to enhanced activation of TBK1 and IRF3.

\section{XPO1 knockdown upregulates p62 expression and enhances type I IFN signaling}

Next, we confirmed XPO1's role in the innate immune response by performing XPO1 knockdown. Similar to KPT-8602 treatment, XPO1 knockdown resulted in p62 nuclear accumulation (Supplementary Fig. 3), increased pIRF3 level, and enhanced KSHV activation of pIRF3 and pTBK1 (Fig. 5A, B). However, XPO1 knockdown did not affect STING protein level. There was no obvious change of LC3B cleavage or p62 level following XPO1 knockdown (Fig. 5B), indicating that XPO1 knockdown did not affect autophagy flux and STING was unlikely degraded by p62mediated autophagy. These results indicated that the enhanced TBK1 and IRF3 activation by XPO1 inhibition were unlikely due to p62's cytosolic function, rather it might be the result of p62 nuclear accumulation.

To determine whether the increased TBK1 and IRF3 activation led to enhanced innate immune responses following XPO1 knockdown, we examined innate immunerelated genes. IFIT1, IFIT2, and IFIT3, which play important roles in KSHV lytic replication ${ }^{41}$. Compared to control cells, XPO1 knockdown significantly increased mRNA levels of IRF7, ISG15, IFIT1, IFIT, and IFIT3 at 48 hpi in KSHV-infected HUVEC (Fig. 5C-H). These results indicated that XPO1 inhibition indeed enhanced innate immune responses. 

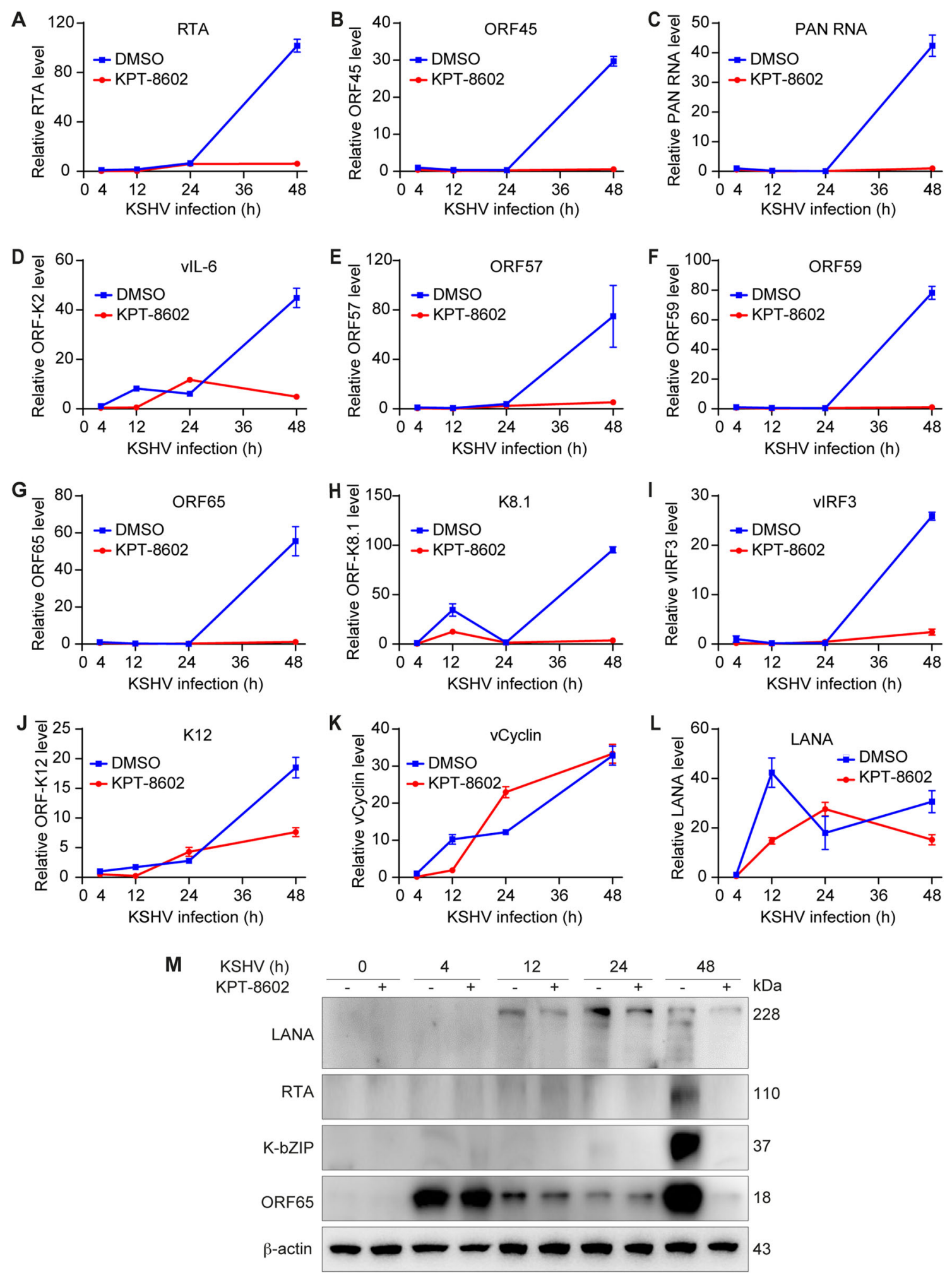

Fig. 2 XPO1 inhibitor KPT-8602 inhibits the expression of KSHV genes except for LANA and vCyclin genes during primary infection. A-L HUVEC were pretreated with $0.5 \mu \mathrm{M} \mathrm{KPT-8602} \mathrm{for} 1 \mathrm{~h}$ and then infected with KSHV in the presence of KPT-8602 for 4, 12, 24, 36, and 48 h. Cells were collected for RNA extraction and examined for the expression of KSHV transcripts by RT-qPCR. M HUVEC were pretreated with $0.5 \mu \mathrm{M} \mathrm{KPT-8602} \mathrm{for} 1 \mathrm{~h}$ and then infected with KSHV in the presence of KPT-8602. The cell lysate was collected at 4, 12, 24, and 48 hpi and analyzed for the expression of viral proteins by Western blotting. $\beta$-actin served as the loading control. 


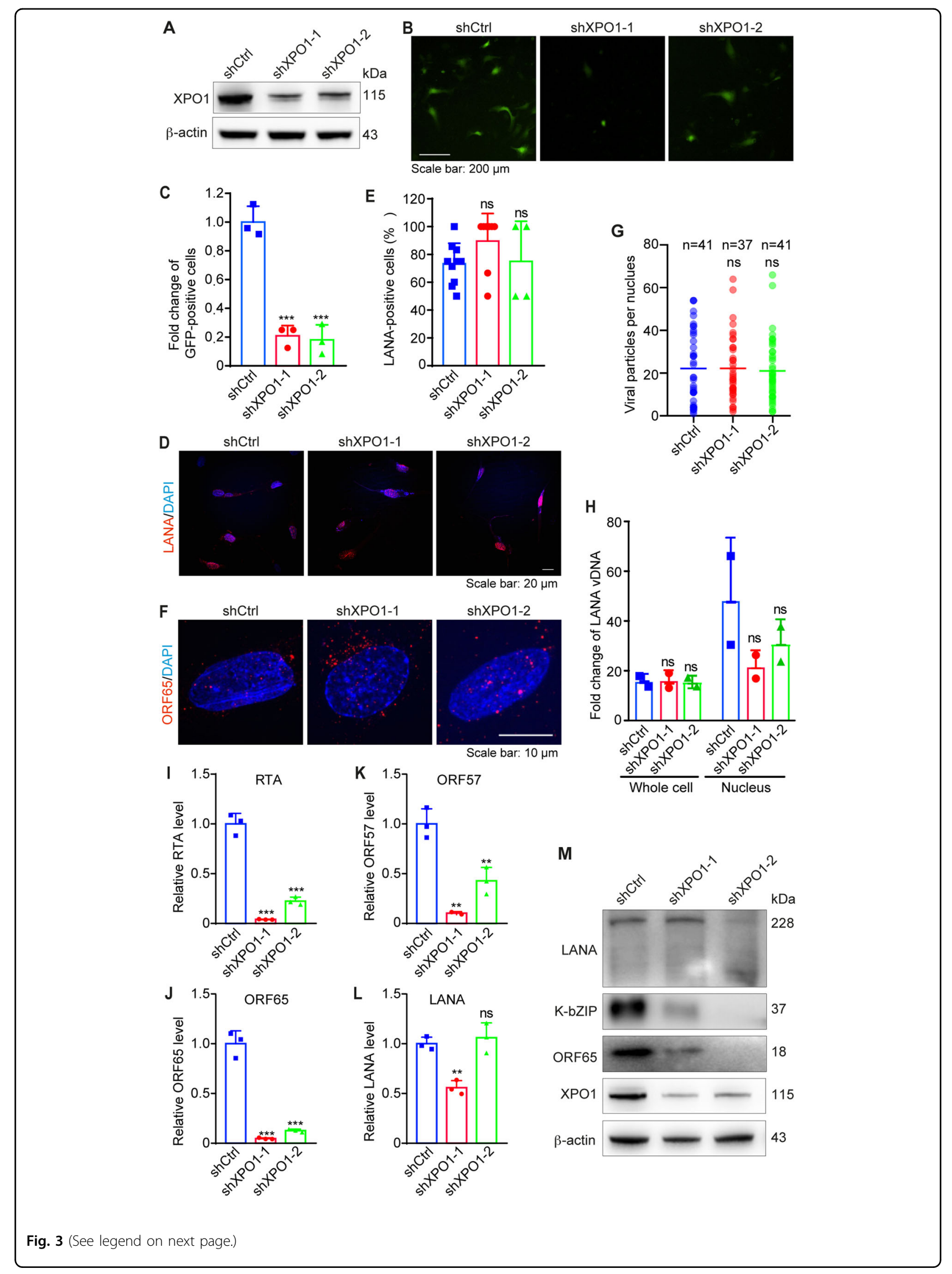


(see figure on previous page)

Fig. 3 XPO1 knockdown blocks the KSHV lytic replication program but has no effect on KSHV infectivity, and virus entry and trafficking during primary infection. A HUVEC were infected with lentiviruses expressing shRNAs targeting XPO1 (shXPO1-1 and shXPO1-2) or control shRNA (shCtrl). Cells were selected with puromycin $(1 \mu \mathrm{g} / \mathrm{ml})$ for $48 \mathrm{~h}$ and analyzed by Western-blotting to monitor the knockdown efficiency. $\beta$-actin was used as the loading control. B, C HUVEC with XPO1 knockdown were infected with KSHV for $96 \mathrm{~h}$. Supernatants were collected and used to titrate infectious virions by infecting fresh HUVEC. At 48 hpi, representative images were taken (B) and used for quantifying the numbers of GFP-positive cells (C). D HUVEC with XPO1 knockdown were infected with KSHV for $48 \mathrm{~h}$. Cells were fixed and stained for LANA (red). The expression level and pattern of LANA are shown. Nuclei were stained with DAPI. Images were taken with a confocal microscopy (magnification $\times 600$ ). E Quantification of LANA-positive cells based on images taken as described in (D). F HUVEC with knockdown of XPO1 were infected with KSHV for 6 h, fixed, and stained for nuclei with DAPI (blue) and KSHV particles with an antibody to ORF65 (red). Images were taken with a confocal microscopy (magnification $\times 1000$ ). G The total number of KSHV particles successfully docked at the perinuclear region of each nucleus was quantified and analyzed based on images taken as described in (F). H HUVEC with XPO1 knockdown infected with KSHV for $6 \mathrm{~h}$ were collected and analyzed for viral genome copy numbers by qPCR using LANA primers. I-L HUVEC with XPO1 knockdown were infected with KSHV and then collected for RNA extraction at 48 hpi. The expression levels of KSHV transcripts were then examined by RT-qPCR. $\mathbf{M}$ HUVEC with XPO1 knockdown were infected with KSHV and collected at 48 hpi. The expression of viral proteins was then analyzed by Western blotting. $\beta$-actin was used as a loading control. *, **, and *** indicate $P$ values of $<$ $0.05,<0.01$, and $<0.001$, respectively; NS not significant.

p62 knockdown restores KSHV lytic replication program but has no effect on virus entry, trafficking, and infectivity

Our results so far showed that XPO1 inhibition reduced KSHV lytic replication by inhibiting the expression of viral lytic genes, which was correlated with p62 nuclear accumulation and enhanced innate immune responses. We further examined p62's role in KSHV primary infection. p62 knockdown had no effect on KSHV infectivity as there was no obvious change in LANA-positive cells with or without KPT-8602 treatment (Fig. 6A-C). p62 knockdown also had no effect on LANA staining pattern and intensity (Fig. 6B), and the number of KSHV particles docked at the perinuclear region (Fig. 6D, E). These results indicated that p62 had no effect on KSHV entry, trafficking, and infectivity. However, p62 knockdown significantly increased mRNA levels of lytic genes RTA, Kb-ZIP, and ORF65 in the presence of KPT-8602, and rescued the inhibitory effect of KPT-8602 on these viral genes (Fig. 6F, H), indicating that p62 regulated expression of KSHV lytic genes. Interestingly, latent gene LANA was also increased, which persisted in the presence of KPT-8602 despite it had no noticeable effect on LANA mRNA (Fig. 6I), suggesting possible different mechanisms regulating KSHV genes following p62 knockdown and KPT-8602 inhibition. p62 knockdown also significantly increased levels of KSHV RTA, Kb-ZIP, and ORF65 proteins (Fig. 6J). Because of KPT-8602's strong inhibitory effect on lytic proteins, the enhanced effect of p62 knockdown was not obvious for RTA and Kb-ZIP but was still visible for ORF65 protein (Fig. 6J). Consistent with the mRNA results, p62 knockdown also slightly increased LANA protein level without KPT8602 treatment albeit it was less consistent with KPT8602 treatment (Fig. 6J). These results indicated that increased p62 nuclear accumulation might at least partially mediate the inhibitory effect of XPO1 inhibition on KSHV lytic genes.

\section{p62 nuclear accumulation induces an innate immune response to block KSHV lytic replication}

To investigate p62's role in innate immune response, we infected HUVEC with KSHV following p62 knockdown. At 48 hpi, p62 knockdown was confirmed (Fig. 7A). p62 knockdown significantly reduced levels of IRF7, ISG15, IFIT1, IFIT2, and IFIT3 genes (Fig. 7B-F), suggesting that p62 was required for maximal activation of the innate immune response during KSHV primary infection.

To explore the role of p62 nuclear accumulation in innate immune response upon XPO1 inhibition, HUVEC with p62 knockdown were pretreated with KPT-8602 at $0.5 \mu \mathrm{M}$ for $1 \mathrm{~h}$ and infected with KSHV for 6 or $12 \mathrm{~h}$. Both pIRF3 and pTBK1 levels were reduced in HUVEC with p62 knockdown compared to cells transduced with the scrambled control (Fig. 8A, B). We observed increased levels of pTBK1, pIRF3, and p62 after KPT-8602 treatment in KSHV-infected HUVEC transduced with the control shRNA. However, p62 knockdown reversed levels of pTBK1 and pIRF3 (Fig. 8C). LC3B cleavage was not increased in HUVEC treated with KPT-8602 (Fig. 8C). These results indicated that p62 nuclear accumulation caused by XPO1 inhibition enhanced TBK1 and IRF3 activation. Finally, RNA was extracted and qRT-PCR was performed at $48 \mathrm{hpi}$ to detect p62 and immune-related genes. Treatment of $0.5 \mu \mathrm{M}$ KPT-8602 increased mRNA levels of p62, IRF7, ISG15, IFIT1, IFIT2, and IFIT3 (Fig. 7D-I). However, p62 knockdown significantly reversed levels of immune-related genes induced by XPO1 inhibition (Fig. 7D-I). Taken together, these results indicated that XPO1 inhibition caused p62 nuclear accumulation, which enhanced type I IFN signaling and blocked KSHV replication.

\section{Discussion}

Viruses often hijack cell machinery to facilitate infection. XPO1 mediates the replication of numerous viruses by regulating the nuclear export of viral and 

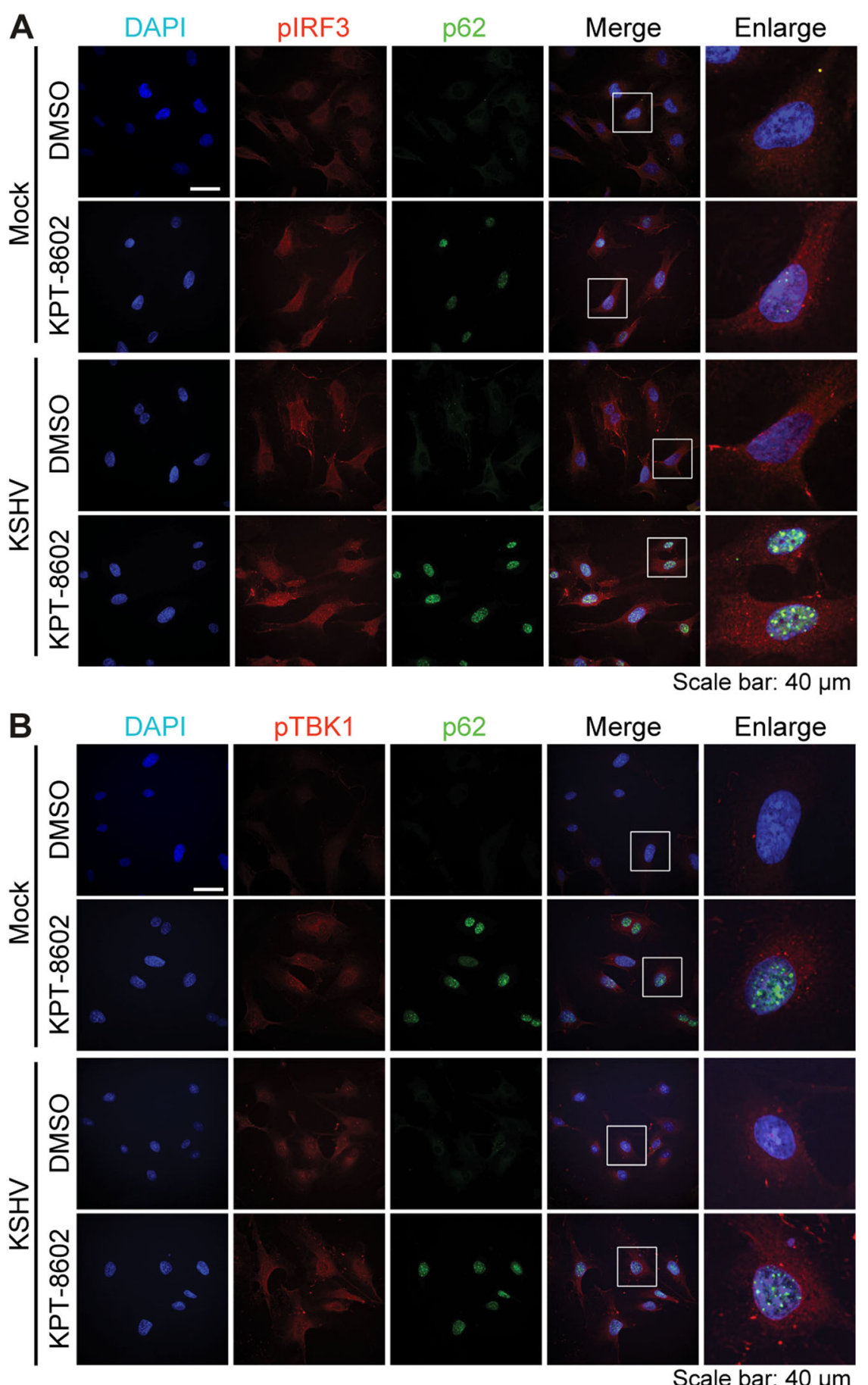

Fig. 4 Treatment with XPO1 inhibitor KPT-8602 induces p62 nuclear retention and enhances IRF3 and TBK1 activation. A, B HUVEC were pretreated with KPT-8602 at $0.5 \mu \mathrm{M}$ or vehicle control DMSO for $1 \mathrm{~h}$ and then infected with KSHV for $12 \mathrm{~h}$ in the presence of KPT-8602 or DMSO. Cells were fixed and co-stained for pIRF3 (A) or pTBK1 (B) together with p62. Nuclei were stained with DAPI. Images were taken with confocal microscopy (magnification $\times 600$ ). Magenta p62 was pseudo-colored to green for better observation.

cellular proteins ${ }^{17,18}$. KSHV LANA2 and ORF45 harbor NESs and are regulated by XPO1 ${ }^{42,43}$. Nuclear shuttling of KSHV proteins could implicate important functions of importins and exportins in KSHV infection.
Nevertheless, XPO1's role in KSHV primary infection remains unknown.

We show that XPO1 inhibition by KPT-8602 or shRNA-mediated knockdown reduces KSHV replication 


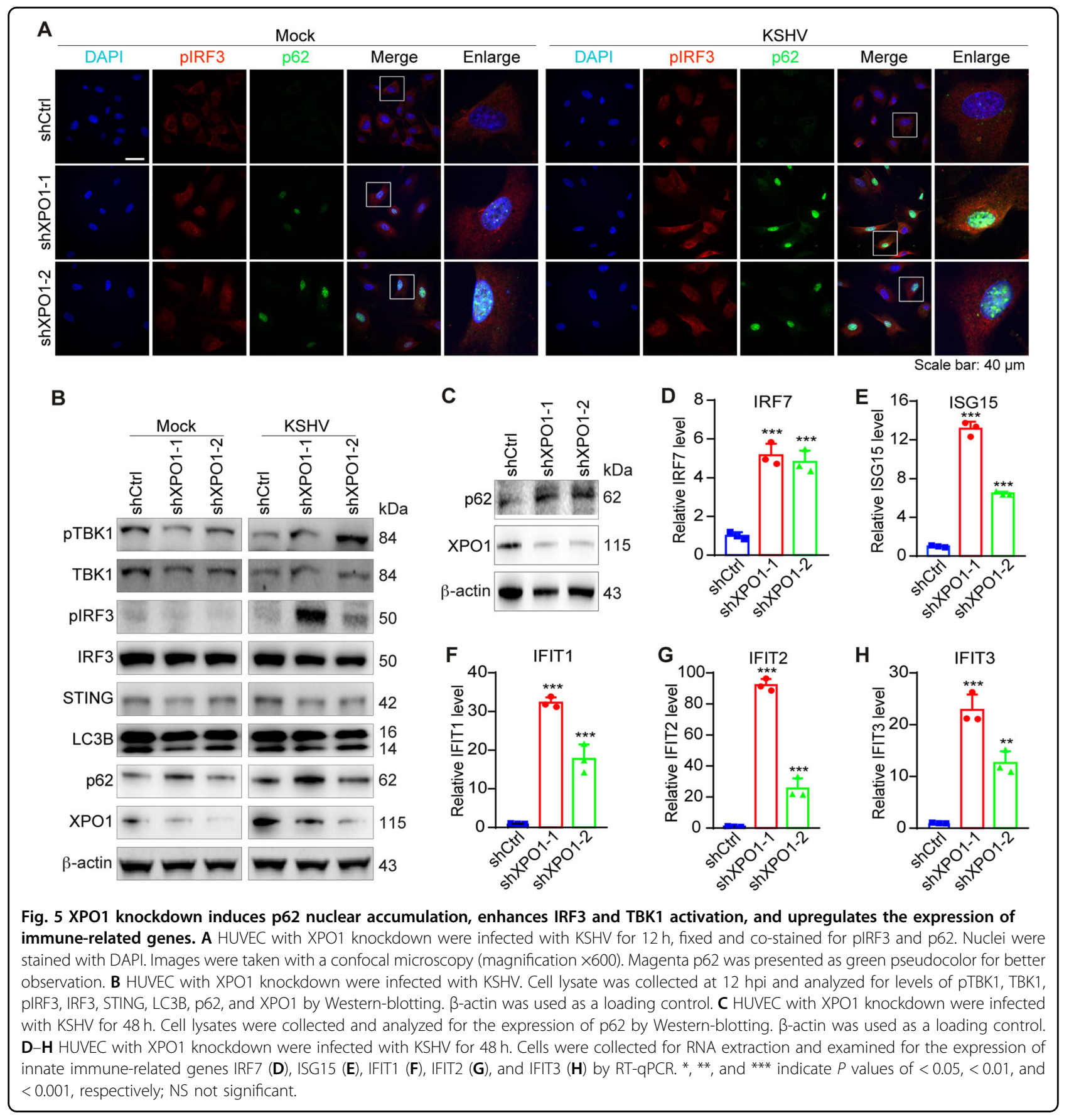

during primary infection without affecting virus entry and trafficking. Numerous XPO1 inhibitors are available including $\mathrm{LMB}$, ratjadone $\mathrm{A}$, thymoquinone, plumbagin, and piperlongumine $^{18,44-48}$. Recently, a novel class of SINE compounds has been identified ${ }^{21-23}$. Unlike LMB, which permanently binds to XPO1 NES groove, SINE compounds such as KPT-330 (selinexor), KPT-335 (verdinexor), KPT185 , and KPT-350 form a reversible covalent bond with the cysteine 528 residue, and hence are less cytotoxic than $\mathrm{LMB}^{21,22,49}$. SINE compounds reduce replication of influenza virus, Venezuelan equine encephalitis virus, and respiratory syncytial virus ${ }^{22,49,50}$. As the second generation of SINE, KPT-8602 manifests improved in vivo efficacy and tolerability in hematologic malignancies compared to KPT$330^{24,51,52}$. We demonstrate KPT-8602 as a promising therapeutic agent against KSHV replication. Furthermore, our previous study has identified KPT-8602 as an effective agent of KSHV-transformed cells ${ }^{31}$.

Our results show that XPO1 inhibition does not affect KSHV entry, trafficking, infectivity, and expression of 


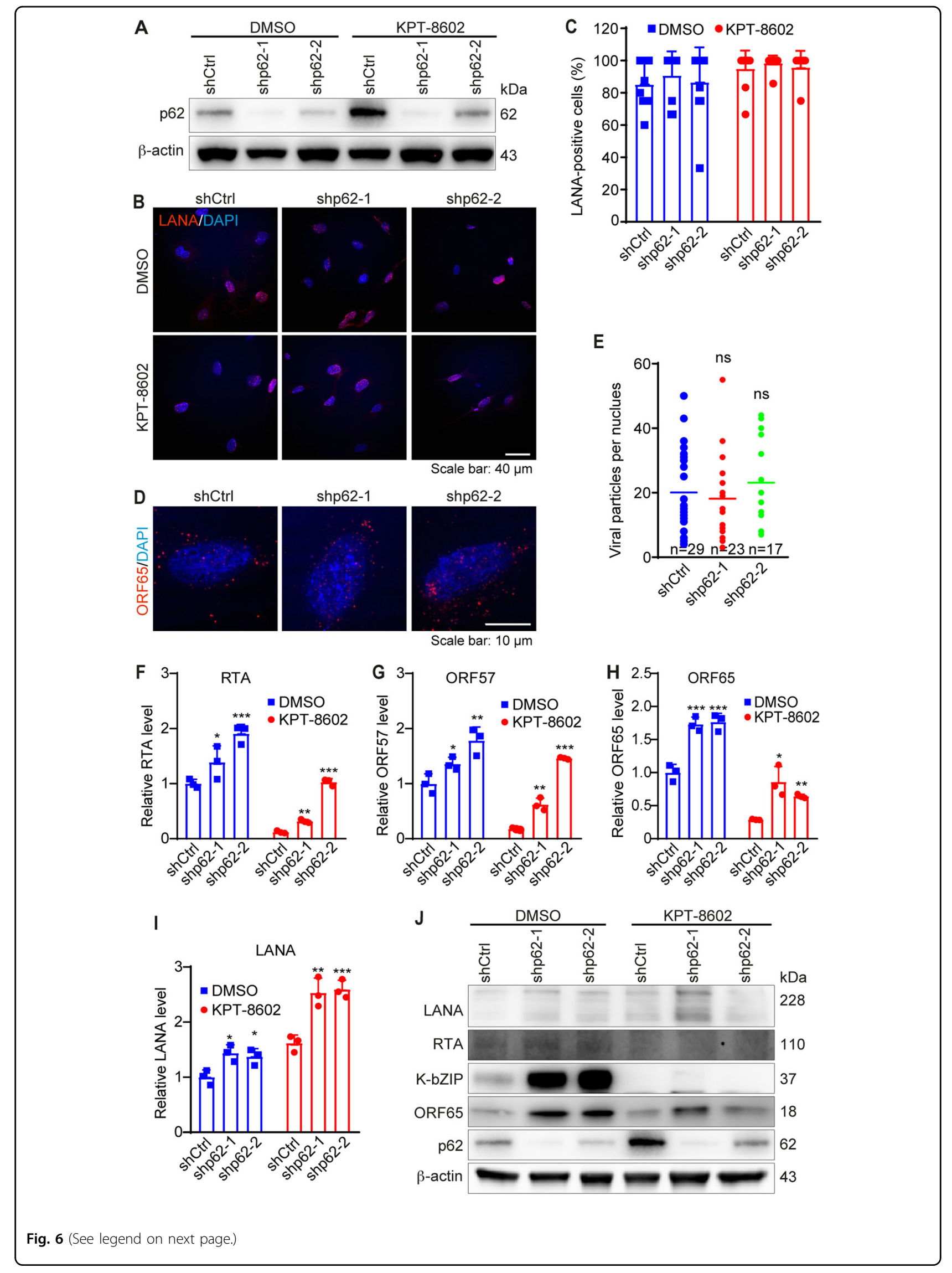


(see figure on previous page)

Fig. 6 p62 knockdown reverses the inhibitory effect of XPO1 inhibitor KPT-8602 on KSHV lytic replication but has no effect on virus infectivity, and entry and trafficking during primary infection. A HUVEC were infected with lentiviruses expressing shRNAs targeting p62 (shp621 and shp62-2) or a control shRNA (shCtrl). Cells were selected with puromycin $(1 \mu \mathrm{g} / \mathrm{ml})$ for $48 \mathrm{~h}$ and analyzed by Western-blotting to monitor the knockdown efficiencies. $\beta$-actin served as the loading control. B HUVEC with p62 knockdown were pretreated with $0.5 \mu M$ KPT-8602. Cells infected with KSHV for $48 \mathrm{~h}$ in the presence of KPT-8602 $(0.5 \mu \mathrm{M})$ were fixed and stained for LANA (red). The expression level and staining pattern of LANA protein are shown. Nuclei were stained with DAPI. Images were taken with a confocal microscopy (magnification $\times 600$ ). C Quantification of LANApositive cells using images taken as described in (B). D HUVEC with p62 knockdown were pretreated with $0.5 \mu \mathrm{M} \mathrm{KPT-8602} \mathrm{for} 1 \mathrm{~h}$, and then infected with KSHV in the presence of KPT-8602 $(0.5 \mu \mathrm{M})$ for $6 \mathrm{~h}$. Cells were fixed and stained for nuclei with DAPI (blue) and KSHV particles with an antibody to ORF65 (red). Images were taken with a confocal microscopy (magnification $\times 1000$ ). E The total number of KSHV particles successfully docked at the perinuclear region of each nucleus was quantified and analyzed based on images taken as described in (D). F-I HUVEC with p62 knockdown was pretreated with $0.5 \mu \mathrm{M} \mathrm{KPT-8602} \mathrm{for} 1 \mathrm{~h}$ and then infected with KSHV in the presence of KPT-8602 $(0.5 \mu \mathrm{M})$. Cells were collected for RNA extraction at $48 \mathrm{hpi}$ and examined for the expression of KSHV genes including RTA (F), ORF57 (G), ORF65 (H), and LANA (I) by RT-qPCR. J HUVEC with p62 knockdown were pretreated with $0.5 \mu \mathrm{M}$ KPT-8602 for $1 \mathrm{~h}$ and then infected with KSHV for $48 \mathrm{~h}$ in the presence of KPT-8602 (0.5 $\mu \mathrm{M})$. Cell lysate was collected and analyzed for the expression of p62 and KSHV proteins by Western-blotting. $\beta$-actin served as the loading control. ${ }^{*},{ }^{* *}$, and ${ }^{* * *}$ indicate $P$ values of $<0.05,<0.01$, and $<0.001$, respectively; NS not significant.

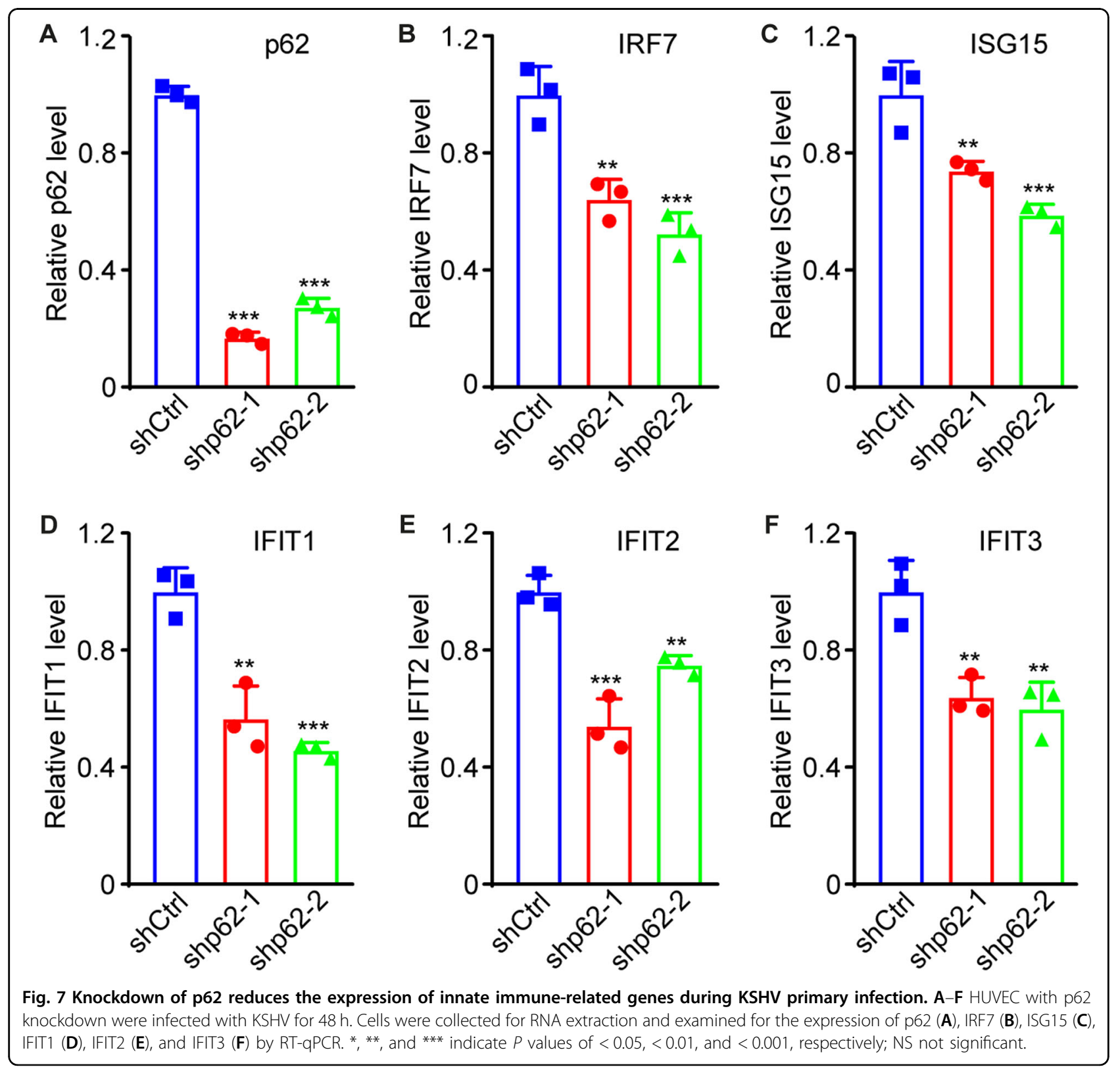




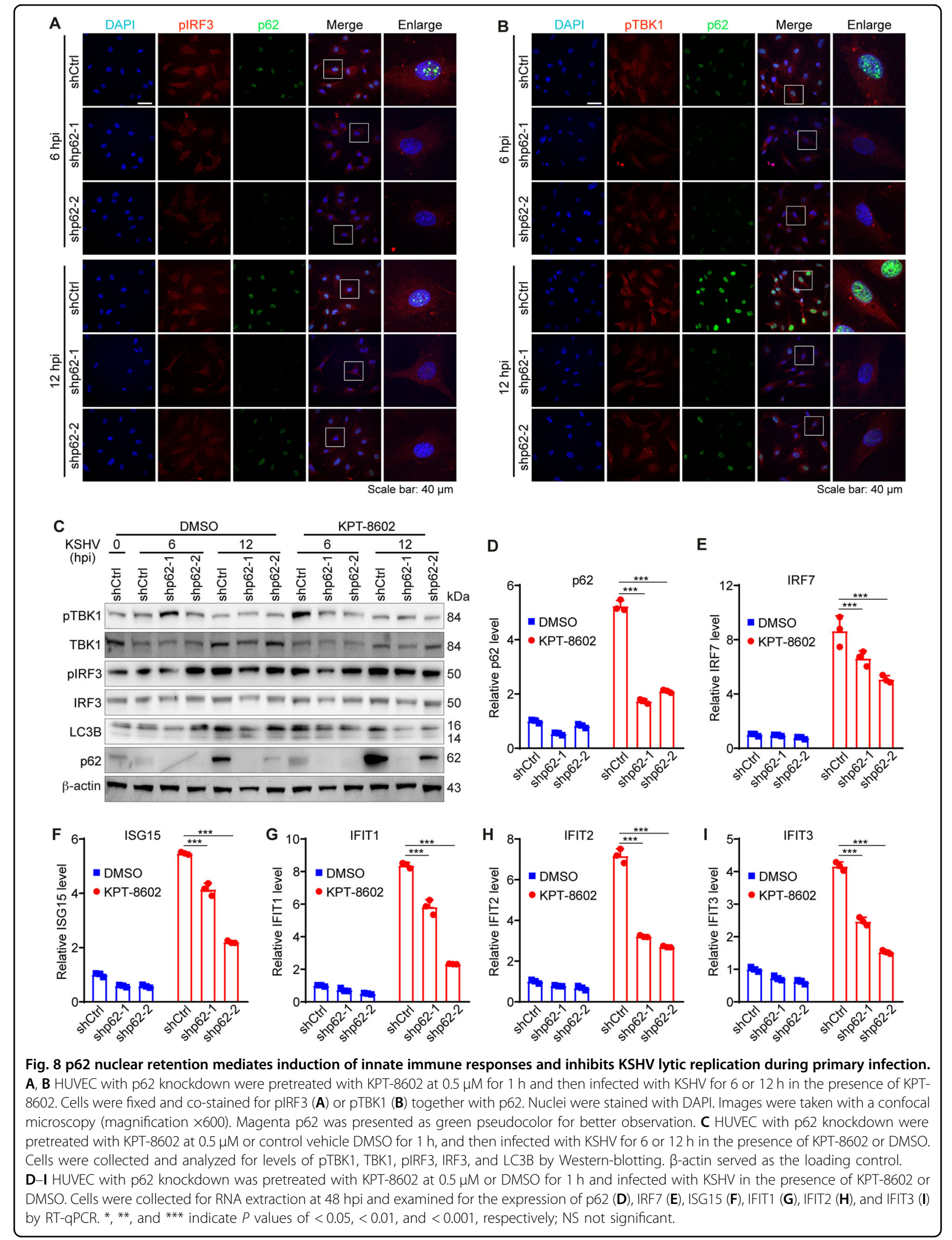


latent gene LANA, rather it inhibits the expression of lytic genes. ORF45 is an IE gene-regulating lytic replication ${ }^{53}$. However, the negative effect of XPO1 inhibition on lytic replication is unlikely due to the inhibition of ORF45 nuclear shuttling or ORF45's direct participation in lytic replication as an expression of RTA, which is required for the expression of other downstream lytic genes including ORF45, precedes that of ORF45 $5^{4}$. Instead, we observe that XPO1 inhibition leads to p62 nuclear accumulation, and enhanced innate immune response accompanying increased expression of innate immune-related genes including IFIT1, IFIT2, and IFIT3 known to inhibit KSHV lytic replication ${ }^{41}$. Nevertheless, since encapsidated ORF45 protein inhibits innate immune response ${ }^{38}$, it remains possible that XPO1 inhibition might affect encapsidated ORF45 protein function, leading to enhanced innate immune responses and decreased KSHV lytic replication.

The effect of KSHV replication on autophagy remains controversial. RTA overexpression induces autophagy in $293 \mathrm{~T}$ cells $^{54}$. While chemical induction of KSHV lytic replication increases autophagy in PEL cells, it is blocked at a late stage of viral replication ${ }^{55}$. Interestingly, KSHV inhibits autophagy by activating mTORC1 during nutrient-deprived conditions, which is important for viral replication ${ }^{56}$. We did not observe any LC3II and p62 changes, indicating that there was no effect on autophagy initiation and flux during the KSHV primary infection of HUVEC. Different cell types and experimental systems likely contribute to these discrepancies.

Autophagy adaptor protein p62 is involved in multiple cellular processes, including autophagy, inflammatory responses, and redox homeostasis ${ }^{57-61}$. p62 dysfunction is associated with Parkinson's disease, Alzheimer's disease, and tumorigenesis ${ }^{62-65}$. Interestingly, p62 is categorized as a new group of innate immunity receptors ${ }^{66}$. p62 delivers specific cytosolic components and ubiquitinated targets to autophagic organelles for removal ${ }^{67,68}$. The role of cytosolic p62 has recently been expanded. p62mediated autophagy selectively degrades RIG-I and STING, attenuating type I IFN signaling ${ }^{32,33}$. p62 harbors active nuclear import and export signals and shuttles continuously between the nucleus and cytoplasm $^{69}$. Nevertheless, p62's role within the nucleus remains unknown. Our results show that XPO1 inhibition enhances the activation of TBK1 and IRF3 and innate immune responses. While p62 nuclear retention following XPO1 inhibition might lead to reduced cytosolic p62 and enhanced innate immune response $\mathrm{e}^{32,33}$, there is no change of STING. Hence, the enhanced innate immune responses following XPO1 inhibition are unlikely due to the compromised cytosolic function of p62, rather it might be due to the gain-of-function of nuclear p62, leading to an enhanced innate immune response and inhibition of KSHV replication. Hence, similar to p62, XPO1 appears to function to control activation of the innate immune response in normal conditions. This function is likely important for controlling hyperactivation of the innate immune system in conditions without pathogen invasion. Indeed, genetic mutations of both p62 and XPO1 are associated with various diseases including inflammatory diseases ${ }^{70-72}$. Hence, precaution should be taken when targeting $\mathrm{p} 62$ and XPO1 for therapy. It remains unclear how XPO1 inhibition and nuclear p62 enhance innate immune responses. Furthermore, p62 and XPO1 might be directly involved in the infection and replication of other viruses, which might warrant further investigations.

\section{Acknowledgements}

We thank members of Dr. Gao's laboratory for technical assistance and helpful discussions. This work was supported by grants from NIH (CA096512,

CA124332, CA132637, CA213275, DE025465, and CA197153) to S.-J. Gao. This work was supported in part by award P30CA047904.

\section{Author contributions}

Wen Meng designed and performed experiments, analyzed and interpreted data, wrote drafts and revised the paper, and approved the final submitted paper. Shou-Jiang Gao secured funding for the project, planned and managed the project, designed experiments, analyzed and interpreted data, wrote and revised the paper, approved the final submitted paper.

Conflict of interest

The authors declare that they have no conflict of interest.

\section{Publisher's note}

Springer Nature remains neutral with regard to jurisdictional claims in published maps and institutional affiliations.

Supplementary Information accompanies this paper at (https://doi.org/ 10.1038/s41419-020-03303-1).

Received: 19 October 2020 Revised: 27 November 2020 Accepted: 2 December 2020

Published online: 04 January 2021

\footnotetext{
References

1. Chang, Y. et al. Identification of herpesvirus-like DNA sequences in AIDSassociated Kaposi's sarcoma. Science 266, 1865-1869 (1994).

2. Cesarman, E., Chang, Y., Moore, P. S., Said, J. W. \& Knowles, D. M. Kaposi's sarcoma-associated herpesvirus-like DNA sequences in AIDS-related bodycavity-based lymphomas. N. Engl. J. Med. 332, 1186-1191 (1995).

3. Soulier, J. et al. Kaposi's sarcoma-associated herpesvirus-like DNA sequences in multicentric Castleman's disease. Blood 86, 1276-1280 (1995).

4. Ye, F., Lei, X. \& Gao, S. J. Mechanisms of Kaposi's sarcoma-associated herpesvirus latency and reactivation. Adv. Virol. 2011, 193860 (2011).

5. Gao, S. J., Deng, J. H. \& Zhou, F. C. Productive lytic replication of a recombinant Kaposi's sarcoma-associated herpesvirus in efficient primary infection of primary human endothelial cells. J. Virol. 77, 9738-9749 (2003).

6. Pan, H., Xie, J., Ye, F. \& Gao, S. J. Modulation of Kaposi's sarcoma-associated herpesvirus infection and replication by MEK/ERK, JNK, and p38 multiple mitogen-activated protein kinase pathways during primary infection. J. Virol. 80, 5371-5382 (2006)

7. Xie, J., Pan, H., Yoo, S. \& Gao, S. J. Kaposi's sarcoma-associated herpesvirus induction of AP-1 and interleukin 6 during primary infection mediated by multiple mitogen-activated protein kinase pathways. J. Virol. 79, 15027-15037 (2005).
} 
8. Yoo, S. M., Zhou, F. C., Ye, F. C., Pan, H. Y. \& Gao, S. J. Early and sustained expression of latent and host modulating genes in coordinated transcriptional program of KSHV productive primary infection of human primary endothelial cells. Virology 343, 47-64 (2005).

9. Grossmann, C. \& Ganem, D. Effects of NFkappaB activation on KSHV latency and lytic reactivation are complex and context-dependent. Virology $\mathbf{3 7 5}$ 94-102 (2008).

10. Krishnan, H. H. et al. Concurrent expression of latent and a limited number of lytic genes with immune modulation and antiapoptotic function by Kaposi's sarcoma-associated herpesvirus early during infection of primary endothelial and fibroblast cells and subsequent decline of lytic gene expression. J. Virol. 78, 3601-3620 (2004).

11. Sadagopan, S. et al. Kaposi's sarcoma-associated herpesvirus induces sustained NF-kappaB activation during de novo infection of primary human dermal microvascular endothelial cells that is essential for viral gene expression. J. Virol. 81, 3949-3968 (2007).

12. Ye, F. C. et al. Kaposi's sarcoma-associated herpesvirus latent gene vFLIP inhibits viral lytic replication through NF-kappaB-mediated suppression of the AP-1 pathway: a novel mechanism of virus control of latency. J. Virol. $\mathbf{8 2}$ 4235-4249 (2008)

13. Hoelz, A., Debler, E. W. \& Blobel, G. The structure of the nuclear pore complex. Annu. Rev. Biochem. 80, 613-643 (2011).

14. Matsuura, Y. Mechanistic insights from structural analyses of Ran-GTPasedriven nuclear export of proteins and RNAs. J. Mol. Biol. 428, 2025-2039 (2016).

15. Moore, M. S. \& Blobel, G. The GTP-binding protein Ran/TC4 is required for protein import into the nucleus. Nature 365, 661-663 (1993).

16. Wente, S. R. \& Rout, M. P. The nuclear pore complex and nuclear transport. Cold Spring Harb. Perspect. Biol. 2, a000562 (2010).

17. Le Sage, V. \& Mouland, A. J. Viral subversion of the nuclear pore complex. Viruses 5, 2019-2042 (2013).

18. Mathew, C. \& Ghildyal, R. CRM1 inhibitors for antiviral therapy. Front. Microbiol. 8, 1171 (2017).

19. Wen, W., Meinkoth, J. L., Tsien, R. Y. \& Taylor, S. S. Identification of a signal for rapid export of proteins from the nucleus. Cell 82, 463-473 (1995).

20. Fornerod, M., Ohno, M., Yoshida, M. \& Mattaj, I. W. CRM1 is an export receptor for leucine-rich nuclear export signals. Cell 90, 1051-1060 (1997).

21. London, C. A. et al. Preclinical evaluation of the novel, orally bioavailable Selective Inhibitor of Nuclear Export (SINE) KPT-335 in spontaneous canine cancer: results of a phase I study. PLOS ONE 9, e87585 (2014).

22. Perwitasari, $\mathrm{O}$. et al. Verdinexor, a novel selective inhibitor of nuclear export, reduces influenza a virus replication in vitro and in vivo. J. Virol. 88 10228-10243 (2014).

23. Ranganathan, $\mathrm{P}$. et al. Preclinical activity of a novel CRM1 inhibitor in acute myeloid leukemia. Blood 20, 1765-1773 (2012)

24. Etchin, J. et al. KPT-8602, a second-generation inhibitor of XPO1-mediated nuclear export, is well tolerated and highly active against AML blasts and leukemia-initiating cells. Leukemia 31, 143-150 (2017).

25. Brulois, K. F. et al. Construction and manipulation of a new Kaposi's sarcomaassociated herpesvirus bacterial artificial chromosome clone. J. Virol. 86, 9708-9720 (2012).

26. Zhou, F. C. et al. Efficient infection by a recombinant Kaposi's sarcomaassociated herpesvirus cloned in a bacterial artificial chromosome: application for genetic analysis. J. Virol. 76, 6185-6196 (2002).

27. Gao, S. J. et al. Seroconversion to antibodies against Kaposi's sarcomaassociated herpesvirus-related latent nuclear antigens before the development of Kaposi's sarcoma. N. Engl. J. Med. 335, 233-241 (1996).

28. Zhao, Q. et al. Kaposi's sarcoma-associated herpesvirus-encoded replication and transcription activator impairs innate immunity via ubiquitin-mediated degradation of myeloid differentiation factor 88. J. Virol. 89, 415-427 (2015).

29. He, M. et al. Cancer angiogenesis induced by Kaposi sarcoma-associated herpesvirus is mediated by EZH2. Cancer Res. 72, 3582-3592 (2012).

30. Greene, W. \& Gao, S. J. Actin dynamics regulate multiple endosomal steps during Kaposi's sarcoma-associated herpesvirus entry and trafficking in endothelial cells. PLoS Pathog. 5, e1000512 (2009).

31. Gruffaz, M. et al. CRISPR-Cas9 screening of Kaposi's sarcoma-associated herpesvirus-transformed cells identifies XPO1 as a vulnerable target of cancer cells. mBio 10, e00866-00819 (2019).

32. Prabakaran, T. et al. Attenuation of CGAS-STING signaling is mediated by a p62/SQSTM1-dependent autophagy pathway activated by TBK1. EMBO J. 37 e97858 (2018)
33. Du, Y. et al. LRRC25 inhibits type I IFN signaling by targeting ISG15-associated RIG-I for autophagic degradation. EMBO J. 37, 351-366 (2018).

34. Ma, Z. et al. Modulation of the CGAS-STING DNA sensing pathway by gammaherpesviruses. Proc. Natl Acad. Sci. USA 112, E4306-E4315 (2015).

35. lqbal, J. et al. Histone H2B-IFl16 recognition of nuclear herpesviral genome induces cytoplasmic interferon-beta responses. PLoS Pathog. 12, e1005967 (2016).

36. Lefort, S., Soucy-Faulkner, A., Grandvaux, N. \& Flamand, L. Binding of Kaposi's sarcoma-associated herpesvirus K-bZIP to interferon-responsive factor 3 elements modulates antiviral gene expression. J. Virol. 81, 10950-10960 (2007).

37. Cloutier, N. \& Flamand, L. Kaposi sarcoma-associated herpesvirus latencyassociated nuclear antigen inhibits interferon (IFN) beta expression by competing with IFN regulatory factor-3 for binding to IFNB promoter. J. Biol. Chem 285, 7208-7221 (2010).

38. Liang, Q. et al. ORF45 of Kaposi's sarcoma-associated herpesvirus inhibits phosphorylation of interferon regulatory factor 7 by IKKepsilon and TBK1 as an alternative substrate. J. Virol. 86, 10162-10172 (2012).

39. Jacobs, S. R. et al. Kaposi's sarcoma-associated herpesvirus viral interferon regulatory factor 1 interacts with a member of the interferon-stimulated gene 15 pathway. J. Virol. 89, 11572-11583 (2015).

40. Zhang, G. et al. Cytoplasmic isoforms of Kaposi sarcoma herpesvirus LANA recruit and antagonize the innate immune DNA sensor cGAS. Proc. Natl Acad. Sci. USA 113, E1034-E1043 (2016).

41. Li, D. \& Swaminathan, S. Human IFIT proteins inhibit lytic replication of KSHV: a new feed-forward loop in the innate immune system. PLoS Pathog. 15, e1007609 (2019).

42. Munoz-Fontela, C. et al. Identification of a nuclear export signal in the KSHV latent protein LANA2 mediating its export from the nucleus. Exp. Cell Res. 311, 96-105 (2005).

43. Li, X. \& Zhu, F. Identification of the nuclear export and adjacent nuclear localization signals for ORF45 of Kaposi's sarcoma-associated herpesvirus. J. Virol. 83, 2531-2539 (2009).

44. Hamamoto, T., Gunji, S., Tsuji, H. \& Beppu, T. Leptomycins A and B, new antifungal antibiotics. I. Taxonomy of the producing strain and their fermentation, purification and characterization. J. Antibiot. 36, 639-645 (1983).

45. Fleta-Soriano, E. et al. The myxobacterial metabolite ratjadone A inhibits HIV infection by blocking the Rev/CRM1-mediated nuclear export pathway. Microb. Cell Fact. 13, 17 (2014).

46. Forouzanfar, F., Bazzaz, B. S. \& Hosseinzadeh, H. Black cumin (Nigella sativa) and its constituent (thymoquinone): a review on antimicrobial effects. Iran. J. Basic Med. Sci. 17, 929-938 (2014)

47. Liu, X. et al. CRM1 is a direct cellular target of the natural anti-cancer agent plumbagin. J. Pharm. Sci. 124, 486-493 (2014).

48. Niu, M. et al. Piperlongumine is a novel nuclear export inhibitor with potent anticancer activity. Chem. Biol. Interact. 237, 66-72 (2015).

49. Lundberg, L. et al. Selective inhibitor of nuclear export (SINE) compounds alter New World alphavirus capsid localization and reduce viral replication in mammalian cells. PLoS Negl. Trop. Dis. 10, e0005122 (2016).

50. Pickens, J. A. \& Tripp, R. A. Verdinexor targeting of CRM1 is a promising therapeutic approach against RSV and influenza viruses. Viruses 10, 48 (2018).

51. Hing, Z. A. et al. Next-generation XPO1 inhibitor shows improved efficacy and in vivo tolerability in hematological malignancies. Leukemia 30, 2364-2372 (2016).

52. Vercruysse, T. et al. The second-generation exportin-1 inhibitor KPT-8602 demonstrates potent activity against acute lymphoblastic leukemia. Clin. Cancer Res. 23, 2528-2541 (2017).

53. Zhu, F. X., Li, X., Zhou, F., Gao, S.-J. \& Yuan, Y. Functional characterization of Kaposi's sarcoma-associated herpesvirus ORF45 by bacterial artificial chromosome-based mutagenesis. J. Virol. 80, 12187-12196 (2006).

54. Wen, H. J., Yang, Z., Zhou, Y. \& Wood, C. Enhancement of autophagy during lytic replication by the Kaposi's sarcoma-associated herpesvirus replication and transcription activator. J. Virol. 84, 7448-7458 (2010).

55. Granato, M. et al. The activation of KSHV lytic cycle blocks autophagy in PEL cells. Autophagy 11, 1978-1986 (2015).

56. Pringle, E. S., Robinson, C. A. \& McCormick, C. Kaposi's sarcoma-associated herpesvirus lytic replication interferes with $\mathrm{MTORC1}$ regulation of autophagy and viral protein synthesis. J. Virol. 93, e00854-00819 (2019).

57. Bjorkoy, G. et al. p62/SQSTM1 forms protein aggregates degraded by autophagy and has a protective effect on Huntingtin-induced cell death. J. Cell Biol. 171, 603-614 (2005). 
58. Pankiv, S. et al. p62/SQSTM1 binds directly to Atg8/LC3 to facilitate degradation of ubiquitinated protein aggregates by autophagy. J. Biol. Chem. 282, 24131-24145 (2007).

59. Lau, A. et al. A noncanonical mechanism of Nrf2 activation by autophagy deficiency: direct interaction between Keap1 and p62. Mol. Cell Biol. 30, 3275-3285 (2010).

60. Zhong, Z. et al. NF-kappaB restricts inflammasome activation via elimination of damaged mitochondria. Cell 164, 896-910 (2016).

61. Carroll, B. et al. Oxidation of SQSTM1/p62 mediates the link between redox state and protein homeostasis. Nat. Commun. 9, 256 (2018).

62. Nakaso, K. et al. Transcriptional activation of p62/A170/ZIP during the formation of the aggregates: possible mechanisms and the role in Lewy body formation in Parkinson's disease. Brain Res. 1012, 42-51 (2004).

63. Duran, A. et al. The signaling adaptor p62 is an important NF-kappaB mediator in tumorigenesis. Cancer Cell 13, 343-354 (2008).

64. Ramesh Babu, J. et al. Genetic inactivation of p62 leads to accumulation of hyperphosphorylated tau and neurodegeneration. J. Neurochem. 106, 107-120 (2008).

65. Mathew, R. et al. Autophagy suppresses tumorigenesis through elimination of p62. Cell 137, 1062-1075 (2009).
66. Deretic, V. Autophagy as an innate immunity paradigm: expanding the scope and repertoire of pattern recognition receptors. Curr. Opin. Immunol. 24, 21-31 (2012).

67. Zheng, Y. T. et al. The adaptor protein p62/SQSTM1 targets invading bacteria to the autophagy pathway. J. Immunol. 183, 5909-5916 (2009).

68. Ponpuak, M. et al. Delivery of cytosolic components by autophagic adaptor protein p62 endows autophagosomes with unique antimicrobial properties. Immunity 32, 329-341 (2010).

69. Pankiv, S. et al. Nucleocytoplasmic shuttling of p62/SQSTM1 and its role in recruitment of nuclear polyubiquitinated proteins to promyelocytic leukemia bodies. J. Biol. Chem. 285, 5941-5953 (2010).

70. Puente, X. S. et al. Whole-genome sequencing identifies recurrent mutations in chronic lymphocytic leukaemia. Nature 475, 101-105 (2011).

71. Bartolome, F. et al. Pathogenic p62/SQSTM1 mutations impair energy metabolism through limitation of mitochondrial substrates. Sci. Rep. 7, 1666 (2017).

72. Cosin-Roger, J. et al. Hypoxia ameliorates intestinal inflammation through NLRP3/mTOR downregulation and autophagy activation. Nat. Commun. 8, 98 (2017). 